\title{
Brain Atrophy Subtypes and the ATN Classification Scheme in Alzheimer's Disease
}

\author{
Nira Cedres ${ }^{a}$ Urban Ekman ${ }^{a}$ Konstantinos Poulakis ${ }^{a}$ Sara Shamsa, b, c \\ Lena Cavallin ${ }^{a, b, c}$ Sebastian Muehlboeck ${ }^{a}$ Tobias Granberg ${ }^{a, b, c}$ \\ Lars-Olof Wahlund $^{a}$ Daniel Ferreira ${ }^{a}$ Eric Westman ${ }^{a} d \quad$ Alzheimer's Disease \\ Neuroimaging Initiative \\ aDivision of Clinical Geriatrics, Department of Neurobiology, Karolinska Institutet, Center for Alzheimer Research, \\ Care Sciences, and Society, Stockholm, Sweden; bepartment of Clinical Neuroscience, Karolinska Institutet, \\ Stockholm, Sweden; 'Department of Neuroradiology, Karolinska University Hospital, Stockholm, Sweden; \\ ${ }^{\mathrm{d} D e p a r t m e n t}$ of Neuroimaging, Institute of Psychiatry, Centre for Neuroimaging Sciences, Psychology and \\ Neuroscience, King's College London, London, UK
}

\section{Keywords}

Alzheimer's disease · Heterogeneity · Subtypes ·

Amyloidosis tauopathy neurodegeneration $\cdot$ Cerebrospinal fluid biomarkers · Factorial analysis

\begin{abstract}
Introduction: We investigated the association between atrophy subtypes of Alzheimer's disease (AD), the ATN classification scheme, and key demographic and clinical factors in 2 cohorts with different source characteristics (a highly selective research-oriented cohort, the Alzheimer's Disease Neuroimaging Initiative [ADNI]; and a naturalistic heterogeneous clinically oriented cohort, Karolinska Imaging Dementia Study [KIDS]). Methods: A total of 382 AD patients were included. Factorial analysis of mixed data was used to investigate associations between AD subtypes based on brain atrophy patterns, ATN profiles based on cerebrospinal fluid biomarkers, and age, sex, Mini Mental State Examination (MMSE), cerebrovascular disease (burden of white matter signal abnormalities, WMSAs), and APOE genotype. Results:
\end{abstract}

Older patients with high WMSA burden, belonging to the typical AD subtype and showing $\mathrm{A}+\mathrm{T}+\mathrm{N}+$ or $\mathrm{A}+\mathrm{T}+\mathrm{N}-$ profiles clustered together and were mainly from ADNI. Younger patients with low WMSA burden, limbic-predominant or minimal atrophy $A D$ subtypes, and $\mathrm{A}+\mathrm{T}-\mathrm{N}-$ or $\mathrm{A}+\mathrm{T}-\mathrm{N}+$ profiles clustered together and were mainly from KIDS. APOE $\varepsilon 4$ carriers more frequently showed the $\mathrm{A}+\mathrm{T}-\mathrm{N}-$ and $\mathrm{A}+\mathrm{T}+\mathrm{N}-$ profiles. Conclusions: Our findings align with the recent framework for biological subtypes of AD: the combination of risk factors, protective factors, and brain pathologies determines belonging of $A D$ patients to distinct subtypes.

(c) 2021 The Author(s)

Published by S. Karger AG, Basel

\section{Background}

Disentangling the biological heterogeneity in Alzheimer's disease (AD) has become an important task in order to guide personalised interventions $[1,2]$. Neuro-

Daniel Ferreira and Eric Westman equal contribution. karger@karger.com www.karger.com/ndd

Karger ${ }^{\prime \prime}$

BOPEN ACCESS (c) 2021 The Author(s)

Published by S. Karger AG, Basel

This is an Open Access article licensed under the Creative Commons Attribution-NonCommercial-4.0 International License (CC BY-NC) (http://www.karger.com/Services/OpenAccessLicense), applicable to the online version of the article only. Usage and distribution for commercial purposes requires written permission.
Correspondence to:

Nira Cedres, nira.cedres@ki.se 
pathological and neuroimaging studies have consistently identified 3 biological subtypes of AD: typical, limbicpredominant, and hippocampal-sparing AD. Typical AD is characterised by a balanced count of neurofibrillary tangles (NFT) or atrophy in the hippocampus and association cortex. Limbic-predominant AD has NFT or atrophy predominantly in the hippocampus. Hippocampal-sparing AD has NFT or atrophy predominantly in the association cortex. Several neuroimaging studies have also identified a fourth subtype with minimal signs of brain atrophy, that is, the minimal atrophy AD subtype $[3,4]$. However, very few studies have investigated the pathophysiological background of these subtypes in vivo [5], which is needed to further disentangle the biological heterogeneity within $\mathrm{AD}$.

Another way to stratify AD patients and also inform on their pathophysiological background is the ATN classification scheme, which is based on dichotomous categories (normal/abnormal) of amyloid-beta (A), tau (T), and neurodegeneration $(\mathrm{N})$ biomarkers. To our knowledge, only one study investigated AD subtypes in combination with ATN profiles, and that study was performed in patients with mild cognitive impairment (MCI) [6].

A task that remains to be done is the incorporation of a category for cerebrovascular disease (CVD, V) to the ATN scheme [7]. White matter signal abnormalities (WMSAs) on magnetic resonance imaging (MRI) are a well-established marker of CVD. WMSAs are implicated in $\mathrm{AD}$ pathogenesis $[8,9]$ and are commonly found in cognitively unimpaired older individuals $[10,11]$. Hence, including the $\mathrm{V}$ dichotomous category in the scheme is important to advance our understanding of associations between amyloid, tau, and vascular pathologies and their contribution to neurodegeneration. Stratifying AD patients into biological subtypes extends the $\mathrm{N}$ category by including a topographical dimension. The topographical dimension likely corresponds to different combinations of amyloid, tau, and vascular and demographic, clinical, and genetic factors. A recent conceptual framework proposed how all these factors interrelate with each other, giving rise to the biological subtypes of $\mathrm{AD}$ [5]. However, this framework has not been tested empirically.

The aim of this study was to investigate the association between AD subtypes, ATN profiles, and key demographic and clinical factors. We evaluated $\mathrm{AD}$ subtypes in combination with ATN profiles in 2 cohorts: a homogeneous research-oriented cohort (the Alzheimer's Disease Neuroimaging Initiative [ADNI]) and a heterogeneous clinically oriented cohort (the KIDS: Karolinska Imaging Dementia Study). Investigating AD subtypes and ATN profiles in cohorts with different characteristics is relevant because these subtypes are thought to result from risk factors, protective factors, and comorbid brain pathologies [5] that are differently represented in researchand clinically oriented cohorts $[5,12]$. We hypothesised that the distribution of $\mathrm{AD}$ subtypes and ATN profiles would differ depending on cohorts and demographic and clinical characteristics. We hypothesised that older patients would include a higher proportion of women with higher WMSA burden, higher proportion of $\mathrm{A}+\mathrm{T}+\mathrm{N}+$ individuals, and lower global cognitive performance; all these related to a higher proportion of individuals classified with typical or limbic-predominant AD subtypes. Younger patients would include a higher proportion of men with lower WMSA burden.

\section{Material and Methods}

\section{Participants}

We combined 2 cohorts of AD patients: ADNI-1 $(N=102)$ [13] and $\operatorname{KIDS}(N=280)$ [14]. ADNI (adni.loni.usc.edu) was launched in 2003 as a public-private partnership led by Principal Investigator Michael W. Weiner, MD. The primary goal of ADNI has been to test whether serial MRI, positron emission tomography, other biological markers, and clinical and neuropsychological assessment can be combined to measure the progression of MCI and early $\mathrm{AD}$. ADNI diagnostic procedures are explained elsewhere [15]. Briefly, patients were clinically diagnosed as AD dementia using the NINCDS-ADRDA criteria for probable AD [16], and they were required to have memory complaints, a Clinical Dementia Rating (CDR) score $\geq 0.5$, significant impairment on activities of daily living, Mini-Mental State Examination (MMSE) scores between 20 and 26, and performance in Logical Memory II of the Wechsler Memory Scale-Revised (WMS-R) $\leq 8$ for 16 years of education, $\leq 4$ for $8-15$ years, and $\leq 2$ for $0-7$ years.

Patients from the KIDS cohort underwent investigation between January 2006 and December 2011. AD diagnosis was determined in multidisciplinary clinical rounds according to the International Statistical Classification of Diseases and Related Health Problems - Tenth Revision (ICD-10), based on all available clinical information (medical history; physical, neurologic, and cognitive examinations; laboratory tests; and brain imaging).

The exclusion criteria in both ADNI and KIDS were other clinical diagnoses (dementia with Lewy bodies, vascular dementia, alcohol-related dementia, MCI, etc.). Further exclusion criteria for the current study were lack of an MRI scan or cerebrospinal fluid (CSF) biomarkers, insufficient MRI scan quality [17], or a history of traumatic brain injury.

Age and sex were included as demographic variables. Clinical severity/global cognition was assessed with the MMSE.

Written informed consent was obtained from all the patients or a legal guardian, in accordance with the Declaration of Helsinki. For ADNI, study protocols were approved by the Institutional Review Boards at each participating centre. For KIDS, ethical approval was obtained from the Regional Ethics Board in Stockholm, Sweden. 


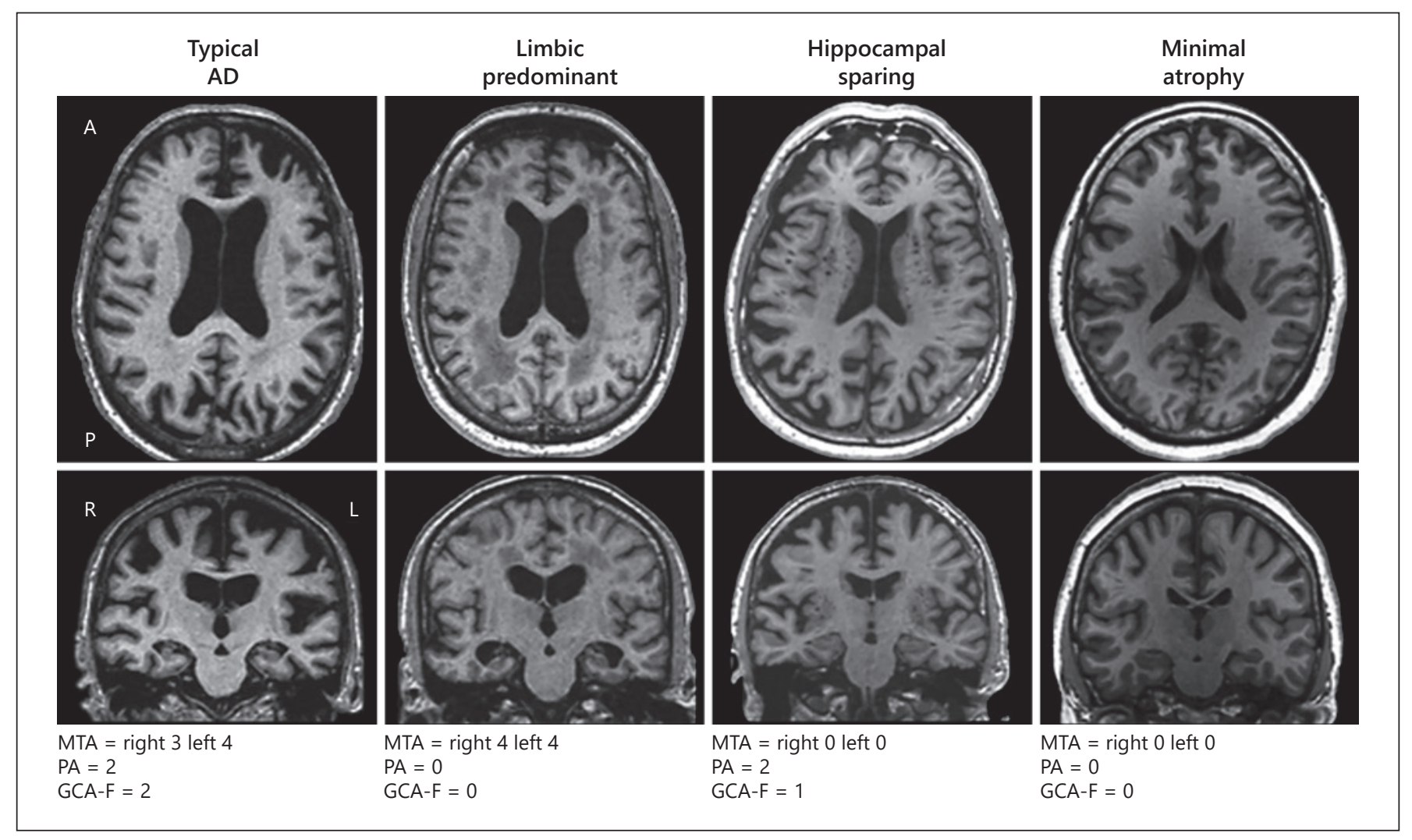

Fig. 1. AD subtypes based on patterns of brain atrophy. Regional atrophy was measured with the MTA, PA, and GCA-F visual rating scales based only on T1-weighted images. In the 3 visual rating scales, a score of zero denotes no atrophy, whereas scores from 1 to 3 (PA and GCA-F) or 4 (MTA) indicate an increasing degree of atrophy. The typical AD subtype was defined as abnormal MTA together with abnormal PA and/or abnormal GCA-F. The limbicpredominant subtype was defined as abnormal MTA alone with

\section{Implementation of the ATN Classification Scheme}

Lumbar puncture for CSF sampling was conducted for both cohorts following standard procedures. For ADNI, amyloid- $\beta_{42}$ $\left(\mathrm{A} \beta_{42}\right)$, phosphorylated-tau (P-tau), and total tau (T-tau) were measured using the multiple xMAP Luminex platform (Luminex Corp, Austin, TX, USA) with INNO-BIA AlzBio3 immunoassay kit-based reagents (Ghent, Belgium; for research use-only reagents) [18]. For KIDS, biomarkers were measured using a sandwich-type ELISA; $A \beta_{42}$ was measured with Innotest b-amyloid (1-42), T-tau with Innotest hTau-Ag, and P-tau with Innotest Phospho-tau (181 P) (Innogenetics, Ghent, Belgium). The unit used for biomarkers is ng/L. All participants were classified into ATN groups according to CSF biomarkers for amyloid- $\beta$ (“A," CSF A $\beta_{42}$ ), tau NFT pathology ("T", CSF P-tau), and unspecific neurodegeneration ("N", CSF Ttau). Each individual was rated as either positive (+; i.e., abnormal) or negative (-; i.e., normal) on each biomarker according to previously published cohort-specific cut-offs: $\leq 192 \mathrm{pg} / \mathrm{mL}$ for A $\beta 42, \geq 23$ $\mathrm{pg} / \mathrm{mL}$ for P-tau, and $\geq 93 \mathrm{pg} / \mathrm{mL}$ for T-tau for the ADNI cohort [18]; and $\leq 550 \mathrm{pg} / \mathrm{mL}$ for $\mathrm{A} \beta_{42}, \geq 80 \mathrm{pg} / \mathrm{mL}$ for P-tau, and $\geq 400 \mathrm{pg} /$ $\mathrm{mL}$ for T-tau for the KIDS cohort [19]. normal PA and GCA-F. The hippocampal-sparing subtype included abnormal PA and/or abnormal GCA-F but normal MTA. The minimal atrophy subtype was defined as normal scores in MTA, PA, and GCA-F. The figure shows examples of each subtype in axial and coronal sections of the brain. AD, Alzheimer's disease; MTA, medial temporal atrophy scale; PA, posterior atrophy scale; GCA-F, global cortical atrophy scale - frontal subscale; A, anterior part of the brain; $\mathrm{P}$, posterior part of the brain; R, right; L, left.

\section{Magnetic Resonance Imaging}

A T1-weighted magnetization-prepared rapid gradient-echo sequence was acquired on ADNI patients (repetition time [RT] 2,300 ms, echo time [ET] 3.6 ms, inversion time [TI] $\sim 1,000 \mathrm{~ms}$, slice thickness $=1.2 \mathrm{~mm}$; http://adni.loni.usc.edu/ methods/mri-tool/mri-analysis/). Data were acquired on $1.5 \mathrm{~T}$ scanners with a voxel size of $1.1 \times 1.1 \times 1.2 \mathrm{~mm}^{3}$. For the KIDS patients, a T1-weighted magnetization-prepared rapid gradientecho sequence $(\mathrm{RT} \sim 1,700 \mathrm{~ms}$, ET $~ 3 \mathrm{~ms}$, TI 1,000 ms, slice thickness $\sim 1.2 \mathrm{~mm}$ ) and a fluid-attenuated inversion recovery sequence $(\mathrm{RT} \sim 8,000 \mathrm{~ms}, \mathrm{ET} \sim 100 \mathrm{~ms}$, TI $=2,100-2,500 \mathrm{~ms}$, slice thickness $\sim 5.0 \mathrm{~mm}$ ) were acquired in $3 \mathrm{MRI}$ scanners at the Radiology Department of the Karolinska University Hospital, Stockholm, Sweden: (1) a 1.5T Magnetom Symphony scanner, (2) 1.5T Magnetom Avanto scanner, and (3) 3T Magnetom Trio scanner [14]. TheHiveDB was used for data management in this study [20].

WMSAs were investigated as a marker of CVD. In the ADNI cohort, WMSAs were assessed through automatically segmented white matter hypointensities from FreeSurfer 6.0.0 (https://surfer. 
Table 1. Cohort characteristics

\begin{tabular}{|c|c|c|c|c|c|c|}
\hline & \multicolumn{3}{|c|}{ (a) Whole cohort } & \multicolumn{3}{|c|}{ (b) A+ subsample } \\
\hline & ADNI & KIDS & $p$ value & ADNI & KIDS & $p$ value \\
\hline$N$ & 102 & 280 & - & 94 & 209 & - \\
\hline Age, years & 75.0 & 67.5 & $<0.001$ & 74.5 & 67.2 & $<0.001$ \\
\hline Sex, \% female & 42 & 58 & 0.008 & 41 & 59 & 0.007 \\
\hline MMSE & 23.5 & 22.0 & 0.005 & 23.5 & 21.8 & 0.002 \\
\hline WMSA burden, $\%$ high & 46 & 20 & $<0.001$ & 45 & 20 & $<0.001$ \\
\hline $\mathrm{A} \beta 42, \%$ abnormal & 92.2 & 76.6 & $<0.001$ & 100 & 100 & - \\
\hline P-tau, \%abnormal & 87.3 & 55.0 & $<0.001$ & 90.4 & 55.5 & $<0.001$ \\
\hline T-tau, \% abnormal & 54.7 & 69.6 & $<0.001$ & 68.1 & 69.4 & 0.927 \\
\hline$A P O E \varepsilon 4, \%$ carriers & 70 & 66 & 0.607 & 76 & 66 & 0.153 \\
\hline
\end{tabular}

MMSE, mini-mental state examination; WMSAs, white matter signal abnormalities; $A \beta$, amyloid $\beta$; P-tau, phosphorylated tau; T-tau, total tau; KIDS, Karolinska imaging dementia study; ADNI, Alzheimer's disease neuroimaging initiative.

nmr.mgh.harvard.edu/). FreeSurfer is increasingly used to automatically segment WMSAs in the form of hypointensities in the T1-weighted MRI sequence [21-25]. In KIDS, the MRI data are clinical, and there is variation across patients in scanning parameters. In such data, the Fazekas visual rating scale [26] is appropriate as a measure of WMSAs because it is not influenced by variation in scanning parameters [27]. Hence, in the KIDS cohort, WMSAs were assessed through the Fazekas scale on fluid-attenuated inversion recovery images. Importantly, hypointense WMSAs from FreeSurfer and the Fazekas scale are strongly associated with each other [28]. We followed a previous study that demonstrated that hypointense WMSAs from FreeSurfer and Fazekas scores can be combined together by converting hypointense WMSA into low and high CVD burden [28]. Hence, we computed a unique WMSA variable by applying a cut-off of 0.00321 on hypointense WMSAs from FreeSurfer after total intracranial volume adjustment. This creates 2 categories of low and high CVD burden that are analogous to Fazekas scores of 0 or 1 defined as low WMSA and Fazekas scores of 2 or 3 defined as high WMSA [28].

Regional brain atrophy was assessed with visual rating scales, as detailed elsewhere [4,29]. Briefly, medial temporal atrophy (MTA) was assessed with Scheltens' scale [30], posterior atrophy (PA) with Koedam's scale [31], and atrophy in the frontal lobe with the global cortical atrophy scale - frontal subscale (GCA-F) [32]. Ratings were performed by an expert neuroradiologist who showed excellent intra- and inter-rater performance: weighted $\kappa$ values for intra-rater reliability: MTA-left $=0.94$, MTA-right $=0.89, \mathrm{PA}=$ 0.88 ; GCA-F $=0.83$; and for inter-rater reliability: MTA-left = $0.71, \mathrm{MTA}$-right $=0.70 ; \mathrm{PA}=0.88, \mathrm{GCA}-\mathrm{F}=0.79$. All ratings were performed blinded to patients' information.

\section{AD Subtypes Based on Patterns of Brain Atrophy}

Deviation from normality in visual ratings was determined using previously published cut-offs [29]. The MTA scores $\geq 1.5, \geq 1.5$, $\geq 2$, and $\geq 2.5$ were considered abnormal for the respective age ranges $45-64,65-74,75-84$, and 85-94 years. Since an age correction does not improve PA and GCA-F diagnostic performance
[29], a score $\geq 1$ was considered abnormal irrespective of the age range [29]. The $3 \mathrm{AD}$ subtypes identified in the previous literature $[33,34]$ were defined based on the combination of MTA, PA, and GCA-F, as in previous studies $[4,6,35,36]$. The minimal atrophy AD subtype $[3,35,37]$ was identified when $\mathrm{AD}$ patients displayed normal scores in MTA, PA, and GCA-F. Visual examples of the 4 $\mathrm{AD}$ subtypes can be found in Figure 1.

\section{Statistical Analysis}

The main aim of this study was to investigate the association between AD subtypes, ATN profiles, and key demographic and clinical factors. Given the nature of our data, which included both continuous and categorical variables, we applied a multivariate method for data analysis called factorial analysis of mixed data (FAMD) [38]. The main strength of FAMD is that it accommodates both quantitative and qualitative data simultaneously. FAMD works as a principal component analysis for quantitative data and as a multiple correspondence analysis for qualitative data [38]. The main objective of FAMD is to simplify multiple data features in latent dimensions (or components), allowing to reduce data and identify association patterns. In our FAMD model, age and MMSE scores were included as continuous variables, and the cohort (ADNI vs. KIDS), ATN categories, AD subtypes, sex (men vs. women), and WMSA burden (high vs. low) were included as categorical variables. A complementary FAMD model was conducted by adding APOE genotype as a categorical variable (carriers of at least $1 \varepsilon 4$ allele vs. non-carriers). One-way ANOVA was used for continuous variables, and the $\chi^{2}$ test was used for categorical data. Missing data on MMSE were estimated via the MissForest algorithm [39] for 6 KIDS patients. All statistical analyses were conducted using R statistical software ( $\mathrm{R}$ Foundation for Statistical Computing, Vienna, http://www-R-project.org). FactoMineR and Factoextra packages were used for FAMD analysis $[40,41]$. $p$ values in all principal and post hoc analyses were adjusted with Benjamini-Hochberg's correction for multiple comparisons [42]. A $p$ value $\leq 0.05$ was deemed statistically significant. 


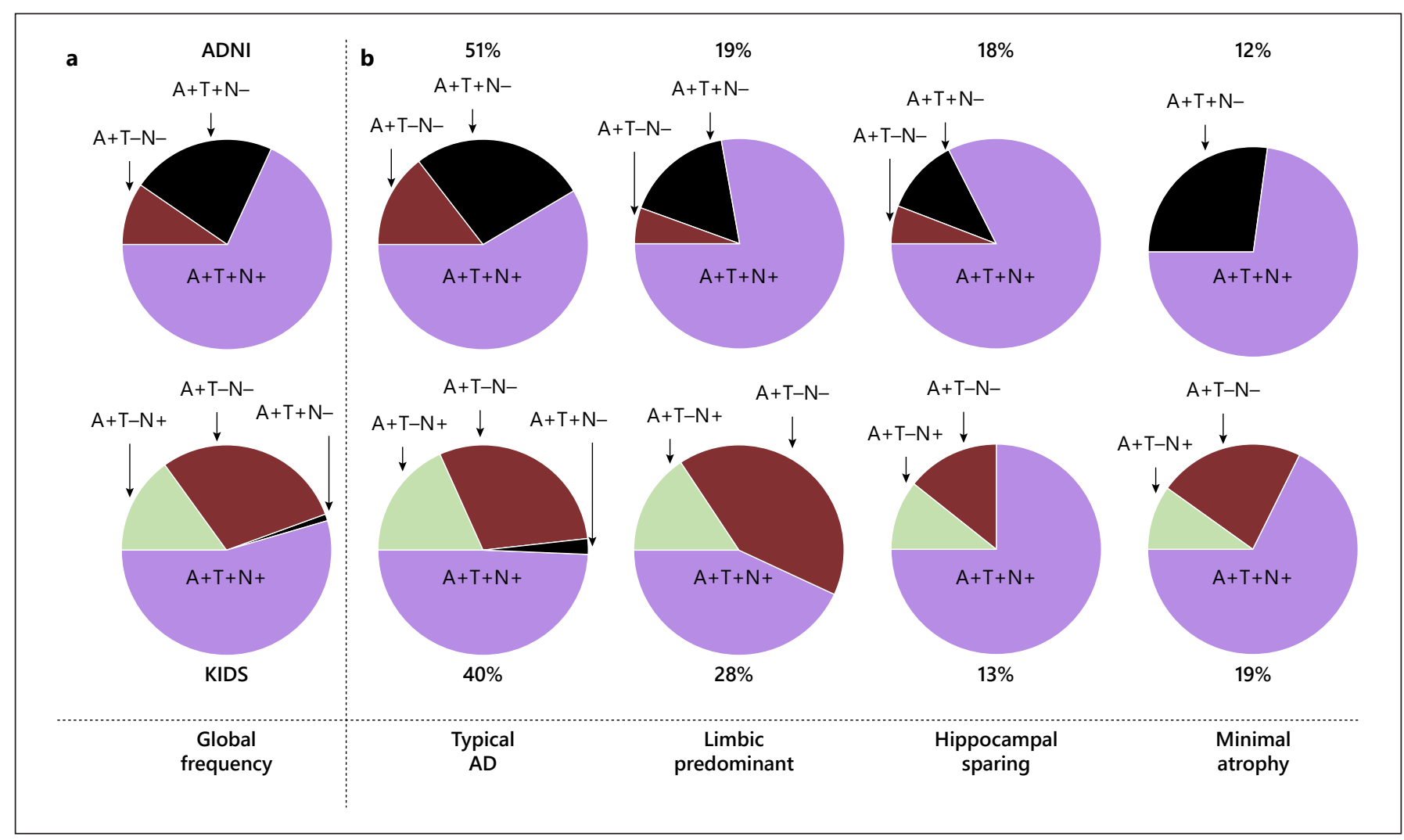

Fig. 2. Frequency of $A T N$ profiles by cohort and AD subtype. a Frequency of ATN profiles within the ADNI (top) and KIDS (bottom) cohorts. b Frequency of ATN profiles across AD subtypes in the ADNI (top) and KIDS (bottom) cohorts. AD, Alzheimer's disease; A-, normal CSF A $\beta$ biomarker; A+, abnormal CSF A $\beta$ biomarker; T-, normal CSF P-tau biomarker; $\mathrm{T}+$, abnormal CSF P-tau biomarker; N-, normal CSF T-tau biomarker; $\mathrm{N}+$, abnormal CSF total tau biomarker; KIDS, Karolinska Imaging Dementia Study; ADNI, Alzheimer's Disease Neuroimaging Initiative; P-tau, phosphorylated tau; T-tau, total tau; CSF, cerebrospinal fluid.

\section{Results}

\section{Cohort Characteristics}

Cohort characteristics are shown in Table $1(N=382)$. ADNI patients were significantly older with higher scores in MMSE and a lower frequency of women as compared with KIDS patients. Further, ADNI patients showed a significantly higher WMSA burden and a higher frequency of abnormal CSF A $\beta 42$ and P-tau levels, while KIDS patients showed a significantly higher frequency of abnormal CSF T-tau levels. Due to the reduced number of amyloid-beta negative $(\mathrm{A}-)$ patients $(N=79), \mathrm{A}$ - groups were excluded from subsequent analyses. The amyloidbeta positive $(\mathrm{A}+)$ subsample is shown in Table $1(N=$ 303). In the A+ subsample, ADNI patients were significantly older with higher scores in MMSE, a higher WMSA burden, and a lower frequency of women and abnormal CSF P-tau levels, as compared with KIDS patients.
Visual inspection of the data shows that typical AD was the most frequent subtype in both ADNI and KIDS (Fig. 2). Limbic-predominant and minimal atrophy AD were more frequent in KIDS, and hippocampal-sparing $\mathrm{AD}$ was more frequent in ADNI (Fig. 2). Minimal atrophy $\mathrm{AD}$ patients were significantly younger than patients from the other subtypes and had a lower WMSA burden than typical AD patients (Table 2). Typical AD patients had worse MMSE scores than the other subtypes. Hippocampal-sparing $\mathrm{AD}$ patients showed a higher proportion of abnormal CSF P-tau levels than limbic-predominant $\mathrm{AD}$ patients.

The frequency of the $\mathrm{A}+\mathrm{T}+\mathrm{N}+$ profile (68\%) was significantly higher $(p<0.001)$ than the frequencies of $\mathrm{A}+\mathrm{T}+\mathrm{N}-(22 \%)$ and $\mathrm{A}+\mathrm{T}-\mathrm{N}-(10 \%)$ profiles in the ADNI cohort (Fig. 2). Interestingly, none of the ADNI patients had an $\mathrm{A}+\mathrm{T}-\mathrm{N}+$ profile. In the KIDS cohort, the frequency of the $\mathrm{A}+\mathrm{T}+\mathrm{N}+$ profile (55\%) was also significantly 
Table 2. Demographic and clinical characteristics by $\mathrm{AD}$ subtype

\begin{tabular}{lccccr}
\hline & Typical AD & $\begin{array}{l}\text { Limbic } \\
\text { predominant }\end{array}$ & $\begin{array}{l}\text { Hippocampal } \\
\text { sparing }\end{array}$ & $\begin{array}{l}\text { Minimal } \\
\text { atrophy }\end{array}$ & $p$ value \\
\hline$N$ & 131 & 76 & 45 & 51 & \\
ADNI & 48 & 18 & 17 & 11 & 0.070 \\
KIDS & 83 & 58 & 28 & 40 & \\
Age, years & $71.6^{\mathrm{d}}$ & $69.8^{\mathrm{d}}$ & $68.5^{\mathrm{d}}$ & $64.4^{\mathrm{a}, \mathrm{b}, \mathrm{c}}$ & $<0.001$ \\
Sex, \% female & 47 & 55 & 64 & 59 & 0.146 \\
MMSE & $20.1^{\mathrm{b}, \mathrm{c}, \mathrm{d}}$ & $23.2^{\mathrm{a}}$ & $23.1^{\mathrm{a}}$ & $23.8^{\mathrm{a}}$ & $<0.001$ \\
WMSA burden, \% high & $37^{\mathrm{d}}$ & 23 & 18 & $14^{\mathrm{a}}$ & 0.015 \\
P-tau, \% abnormal & 64 & $55^{\mathrm{c}}$ & $82^{\mathrm{b}}$ & 75 & 0.019 \\
T-tau, \% abnormal & 64 & 63 & 84 & 77 & 0.041 \\
APOE $\varepsilon 4, \%$ carrier & 76 & 68 & 56 & 72 & 0.210 \\
\hline
\end{tabular}

None of the paired comparisons for AD subtypes in the T-tau measure was significant after the Benjamini-Hochberg correction for multiple comparisons. MMSE, mini-mental state examination; WMSAs, white matter signal abnormalities; P-tau, phosphorylated tau; T-tau, total tau; AD, Alzheimer's disease; KIDS, karolinska imaging dementia study; ADNI, Alzheimer's disease neuroimaging initiative. ${ }^{\text {a }}$ Significantly different from typical AD. ${ }^{b}$ Significantly different from limbic predominant. ${ }^{c}$ Significantly different from hippocampal sparing. ${ }^{\mathrm{d}}$ Significantly different from minimal atrophy. higher $(p<0.001)$ than the other ATN profiles. Interestingly, we observed a substantial proportion of $\mathrm{A}+\mathrm{T}-\mathrm{N}+$ (15\%) patients in the KIDS cohort. The $\mathrm{A}+\mathrm{T}-\mathrm{N}-$ profile accounted for $30 \%$ of the KIDS patients, and the A+T+Nprofile included less than $1 \%$ of KIDS patients.

\section{Association between AD Subtypes, ATN Profiles, and \\ Key Demographic and Clinical Factors}

Visual inspection of the correspondence between $\mathrm{AD}$ subtypes and ATN profiles showed that in ADNI, the $\mathrm{A}+\mathrm{T}+\mathrm{N}+$ was the most frequent group across all $\mathrm{AD}$ subtypes (Fig. 2). The A+T-N-group was present in every subtype but in minimal atrophy $\mathrm{AD}$ in ADNI. In KIDS, the frequency of $\mathrm{A}+\mathrm{T}+\mathrm{N}+$ was the lowest in typical and limbic-predominant $\mathrm{AD}$ (Fig. 2). In contrast, $\mathrm{A}+\mathrm{T}-\mathrm{N}+$ was equally distributed across all AD subtypes. The $\mathrm{A}+\mathrm{T}+\mathrm{N}-$ group was only present in the typical AD subtype in KIDS. These visual analyses were further supported by the FAMD models, also showing the correspondence among AD subtypes, ATN profiles, WMSA burden, age, sex, MMSE, cohort, and APOE genotype.

The main FAMD model $(N=382)$ showed 3 dimensions that explained $42.3 \%$ of the variance (Table 3 ). Dimension 1 explained $18.6 \%$ of the variance and was mainly driven by cohort and age (although WMSA burden, ATN, AD subtype, and sex also contributed statistically significantly to dimension 1). Dimension 2 explained $12.2 \%$ of the variance and was driven by AD subtype (although MMSE, ATN, and cohort also contributed statis-
Table 3. Contribution of each variable to the dimensions of the FAMD

\begin{tabular}{lccc}
\hline & $\begin{array}{c}\text { Dimension 1 } \\
(\mathrm{R} 2=18.6 \%)\end{array}$ & $\begin{array}{c}\text { Dimension 2 } \\
(\mathrm{R} 2=12.2 \%)\end{array}$ & $\begin{array}{c}\text { Dimension 3 } \\
(\mathrm{R} 2=11.5 \%)\end{array}$ \\
\hline Age, years & 25.3 & 1.1 & 2.5 \\
MMSE & 1.0 & 25.4 & 30.1 \\
Cohort & 28.9 & 8.2 & 0.9 \\
Subtypes & 7.9 & 42.1 & 31.9 \\
ATN & 14.1 & 17 & 29.6 \\
WMSAs & 18.2 & 3.1 & 3.2 \\
Sex & 4.7 & 3.1 & 1.8 \\
\hline
\end{tabular}

Values represent the percentage of contribution of each variable to the total variation captured by each dimension. MMSE, mini-mental State examination; WMSA, white matter signal abnormalities; FAMD, factorial analysis of mixed data.

tically significantly to dimension 2). Dimension 3 explained $11.5 \%$ of the variance and was driven by AD subtype, MMSE, and ATN.

Although ATN and AD subtypes contributed to the 3 dimensions, it was different categories within ATN and $\mathrm{AD}$ subtype that differently contributed to the dimensions. To elaborate on this, dimensions 2 and 3 were plotted against dimension 1 . Figure 3 shows that older patients have higher WMSA burden and tend to be from the ADNI cohort, clustering together. This cluster also showed a high frequency of $\mathrm{A}+\mathrm{T}+\mathrm{N}+$ and $\mathrm{A}+\mathrm{T}+\mathrm{N}-$ profiles, and a 


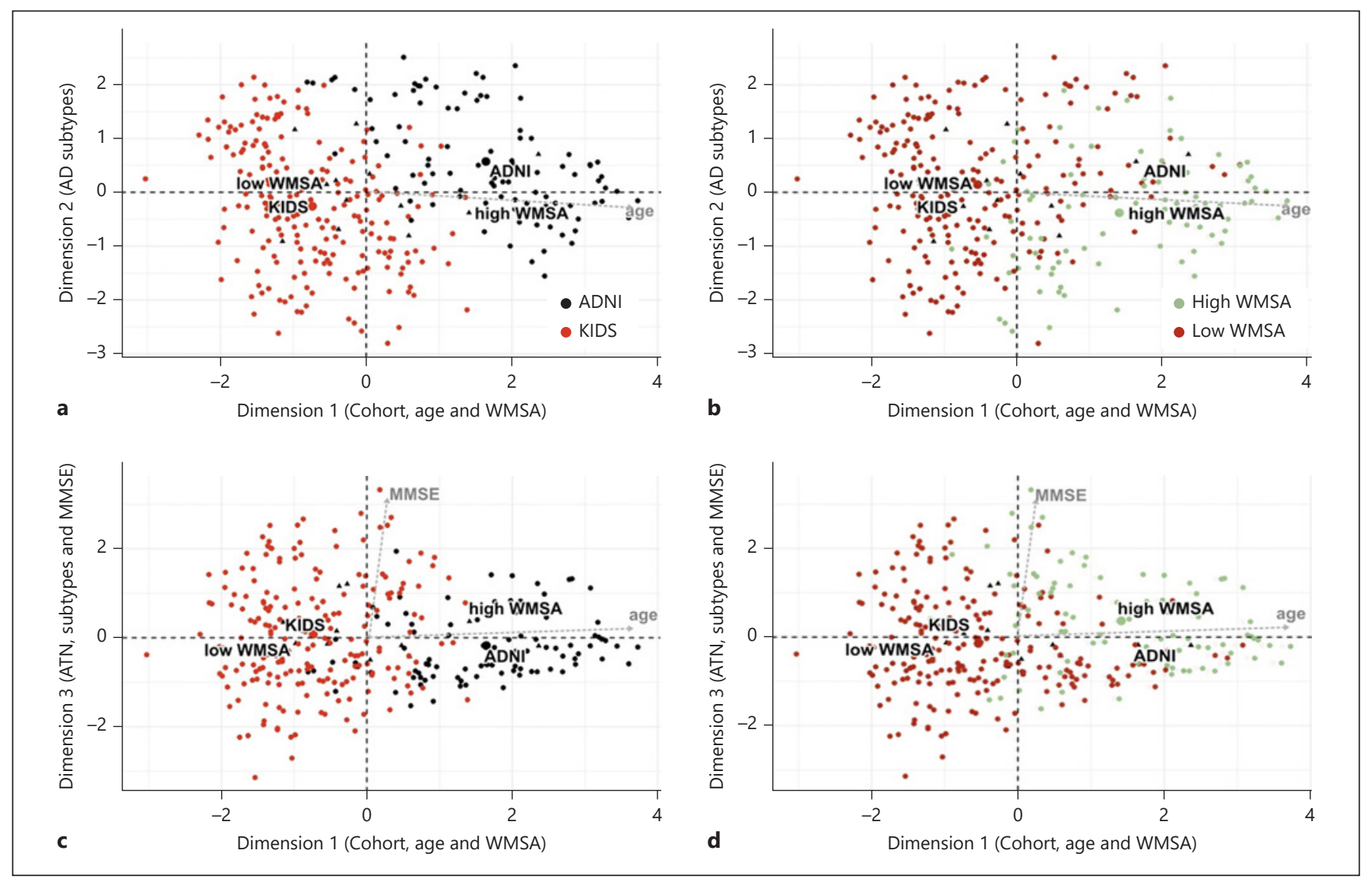

Fig. 3. FAMD: scatterplots for cohort and WMSA burden. Dots represent individual AD patients. In a-d, the $x$ axis represents dimension 1 . In $\mathbf{a}, \mathbf{b}$, the $y$-axis represents dimension 2 . In $\mathbf{c}, \mathbf{d}$, the $y$-axis represents dimension 3. AD, Alzheimer's disease; WMSA, white matter signal abnormalities; A +, CSF A $\beta$ abnormal; T-, CSF P-tau normal; T+, CSF P-tau abnormal; N-, CSF T-tau normal; N+, T-tau abnormal; MMSE, Mini-Mental State Examination; FAMD, factorial analysis of mixed data; P-tau, phosphorylated tau; T-tau, total tau; CSF, cerebrospinal fluid.

high frequency of patients with the typical $\mathrm{AD}$ subtype (Fig. 4). This cluster also includes a substantial proportion of patients with the hippocampal-sparing $\mathrm{AD}$ subtype. However, the AD subtype factor is slightly oblique to dimension 1; hence, many hippocampal-sparing $\mathrm{AD}$ patients fall within a second cluster including younger patients with lower WMSA burden who tended to be from the KIDS cohort (Fig. 3). This second cluster showed a high frequency of $\mathrm{A}+\mathrm{T}-\mathrm{N}-$ and $\mathrm{A}+\mathrm{T}-\mathrm{N}+$ profiles (Fig. 4) and included most of the patients with limbic-predominant and minimal atrophy AD. However, as explained previously, the $\mathrm{AD}$ subtype factor is slightly oblique to dimension 1, so this second cluster included patients from typical and hippocampal-sparing $\mathrm{AD}$ subtypes as well.

Dimension 3 separates the AD subtypes and ATN profiles more clearly and shows the effect of MMSE. When dimension 3 is plotted against dimension 1, it can be observed that $\mathrm{A}+\mathrm{T}+\mathrm{N}+$ and $\mathrm{A}+\mathrm{T}+\mathrm{N}-$ have lower MMSE scores, independent of the cohort, WMSA burden, and age (Fig. 4). Limbic-predominant $\mathrm{AD}$ patients have higher MMSE scores, and typical and hippocampal-sparing AD patients have lower MMSE scores, while minimal atrophy $\mathrm{AD}$ does not completely align with MMSE scores (Fig. 4).

The complementary FAMD model adding APOE $\varepsilon 4$ status was conducted in the subsample with available APOE data $(N=178)$. This model showed very similar results to the main FAMD model. Dimension 1 explained $19 \%$ of the variance and was mainly driven by cohort and age. Dimension 2 explained 12\% of the variance and was driven by $\mathrm{AD}$ subtype. Dimension 3 explained $11 \%$ of the variance and was driven by ATN and APOE \&4 status. 


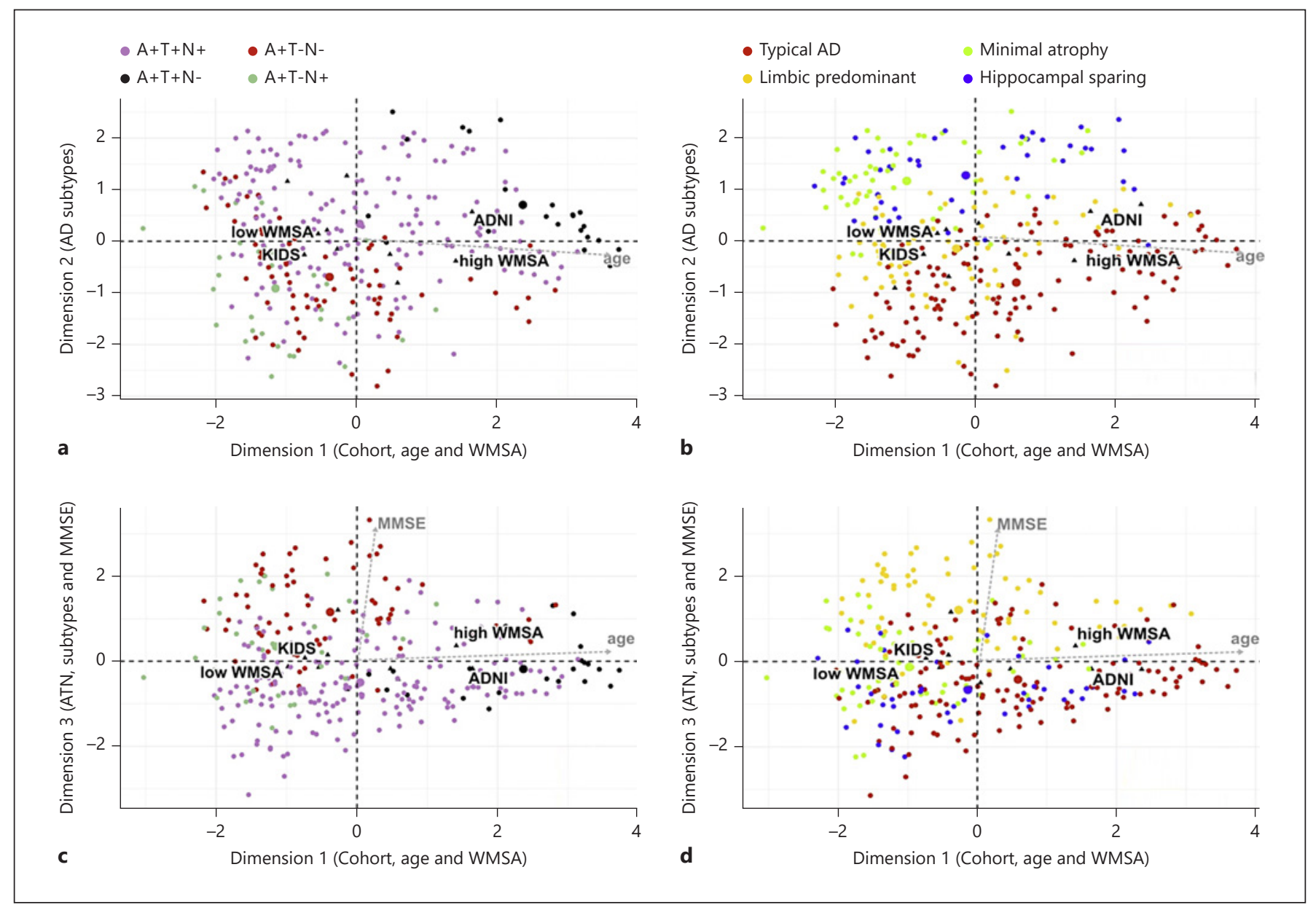

Fig. 4. FAMD: scatterplots for $A D$ subtypes and $A T N$ profiles. Dots represent individual AD patients. In a-d, the $x$-axis represents dimension 1 . In $\mathbf{a}, \mathbf{b}$, the $y$-axis represents dimension 2 . In $\mathbf{c}, \mathbf{d}$, the $y$-axis represents dimension 3. AD, Alzheimer's disease; WMSAs, white matter signal abnormalities; A+, CSF A $\beta$ abnormal; T-, CSF P-tau normal; T+, CSF P-tau abnormal; N-, CSF T-tau normal; N+, T-tau abnormal; MMSE, Mini-Mental State Examination; FAMD, factorial analysis of mixed data; P-tau, phosphorylated tau; T-tau, total tau; CSF, cerebrospinal fluid.

Within dimension 3, APOE $\varepsilon 4$ carriers showed a higher frequency of $\mathrm{A}+\mathrm{T}-\mathrm{N}-$ and $\mathrm{A}+\mathrm{T}+\mathrm{N}-$ profiles and a tendency to include patients with typical AD.

\section{Discussion}

We investigated the association between $\mathrm{AD}$ subtypes and ATN classification scheme in 2 cohorts with different source characteristics. As hypothesised, the distribution of AD subtypes and ATN profiles differed between the research-oriented cohort (i.e., ADNI) and the clinically oriented cohort (i.e., KIDS). In addition, we empirically tested the recent conceptual framework of biological sub- types of $\mathrm{AD}$ [5]. We applied a multivariate method for data analysis to investigate the association between $\mathrm{AD}$ subtypes, ATN profiles, and key demographic and clinical factors, including WMSA burden, age, sex, global cognition, and APOE genotype. To our knowledge, this study is the first in investigating $\mathrm{AD}$ subtypes in combination with ATN profiles in patients with AD dementia.

The recent conceptual framework of biological subtypes of $\mathrm{AD}$ proposes 2 dimensions: severity and typicality [5]. The severity dimension corresponds to the " $\mathrm{N}$ " domain of the ATN scheme and includes typical and minimal $\mathrm{AD}$ as the 2 extremes of a continuum of neurodegeneration. Typical $\mathrm{AD}$ is in the severe end of the continuum, and minimal atrophy AD is in the other end. In our study, 
typical $\mathrm{AD}$ was the most frequent subtype in $\mathrm{ADNI}$ and KIDS. This result might seem unexpected since ADNI recruited mild to moderate $\mathrm{AD}$ patients, while typical $\mathrm{AD}$ would reflect full-blown $\mathrm{AD}$ at the highest degree of neurodegeneration. However, ADNI is a highly selective research cohort with strict inclusion criteria [12] that aimed to recruit the prototypical amnestic presentation of $\mathrm{AD}$, which correlates with the typical AD subtype in neuropathological studies [33]. In addition, ADNI recruited patients with high education, which probably positively influenced patients' cognitive reserve, possibly explaining why patients in ADNI have overt ATN and brain atrophy profiles, yet they are at mild to moderate clinical stages. On the other hand, the clinically oriented KIDS cohort is a naturalistic memory clinic sample that includes younger patients mainly at an early clinical stage with challenging differential diagnoses. This could explain the higher frequency of patients in the minimal atrophy AD subtype in KIDS.

The typicality dimension in the conceptual framework of biological subtypes of $\mathrm{AD}$ includes limbic-predominant $\mathrm{AD}$ on the one side, and hippocampal-sparing $\mathrm{AD}$ on the opposite side, both deviating from typical $\mathrm{AD}$ in the middle [5]. We found that limbic-predominant AD was slightly more frequent in KIDS, and hippocampalsparing $\mathrm{AD}$ was slightly more frequent in ADNI. Based on previous studies [5], we hypothesised that these differences could be explained by demographic and clinical factors. To further test this hypothesis, we investigated the association among the AD subtype, ATN profiles, age, sex, cognitive status, and APOE genotype (discussed below).

$\mathrm{A}+\mathrm{T}+\mathrm{N}-$ and $\mathrm{A}+\mathrm{T}+\mathrm{N}+$ profiles were more frequent in ADNI than in KIDS. The current biological definition of $\mathrm{AD}$ [43] postulates that $\mathrm{A}+$ is the first pathological change, followed by $\mathrm{T}+$ and, eventually, $\mathrm{N}+$. Further, $\mathrm{A}+$ and $\mathrm{T}+$ reflect $\mathrm{AD}$ pathology, while $\mathrm{N}+$ is unspecific, with pathologies other than $\mathrm{A}$ and $\mathrm{T}$ contributing to neurodegeneration $(\mathrm{N})$ as well. Hence, our finding of $\mathrm{A}+\mathrm{T}+\mathrm{N}-$ and $\mathrm{A}+\mathrm{T}+\mathrm{N}+$ being more frequent in ADNI than in KIDS could be related to the stricter selection criteria of ADNI, with a special interest on the amnestic form of AD. This interpretation is further supported by our finding of a high frequency of $\mathrm{A}+\mathrm{T}-\mathrm{N}+$ in $\mathrm{KIDS}$. The $\mathrm{N}+$ category in the presence of a $\mathrm{T}$ category suggests that the neurodegeneration in these patients is due to some pathology other than tau NFT, which suggests a mixed aetiology of clinical AD. As explained earlier, KIDS is a heterogeneous naturalistic memory clinic sample including young patients with challenging diagnoses, as reflected by the higher frequency of the $\mathrm{A}+\mathrm{T}-\mathrm{N}+$ profile. Hence, the frequency of ATN profiles is highly dependent upon cohort, but not so much upon the AD subtype.

CVD could be one of the non-AD pathologies contributing to $\mathrm{N}+$. A previous study demonstrated that CVD contributes differently to AD subtypes [36]. Our current study provides novel data on the association between CVD, AD subtype, and ATN classification scheme. We found a higher WMSA burden in ADNI. This result may be unexpected since vascular risk factors (a predictor of WMSA) [44, 45] are exclusion criteria in ADNI. However, previous studies showed that WMSA burden increases with older age $[45,46]$, and ADNI patients are older than KIDS patients in our study, which could explain our finding of higher WMSA in the ADNI. This finding aligns with the recent conceptual framework of biological subtypes of $\mathrm{AD}$ [5], that is, older patients had higher WMSA burden, they more frequently had an $\mathrm{A}+\mathrm{T}+\mathrm{N}+$ profile, and included a higher proportion of typical and limbic-predominant AD cases. Further, typical $\mathrm{AD}$ patients showed greater cognitive impairment than limbic-predominant AD [5].

Our complementary FAMD model showed that $A P O E$ $\varepsilon 4$ carriers tended to cluster together with patients with $\mathrm{A}+\mathrm{T}-\mathrm{N}-$ and $\mathrm{A}+\mathrm{T}+\mathrm{N}-$ profiles who belonged to the typical AD subtype. The association between the APOE $\varepsilon 4$ genotype and amyloid-beta pathology $(\mathrm{A}+)$ is a well-established finding [47]. Further, previous studies showed that the frequency of $A P O E \& 4$ is higher in typical $A D$ than in hippocampal-sparing AD [5]. Sex only marginally contributed to dimension 1 in the main FAMD model. Although sex is also listed as one of the contributors to the emergence of $\mathrm{AD}$ subtypes [5], our current data suggest that the contribution of sex is less prominent than that of ATN profiles and other demographic and clinical factors. All in all, our findings largely support the recent conceptual framework of biological subtypes of AD [5].

The AD subtype and ATN classifications are 2 popular approaches to disentangle disease heterogeneity in $\mathrm{AD}$. An important finding in our study is that the correspondence between AD subtypes and ATN profiles is not absolute, suggesting that both approaches may capture complementary information. The FAMD model showed that the AD subtype was the main driver of one of the dimensions (dimension 2), while ATN always emerged as a secondary driver after the AD subtype, MMSE, cohort, age, or WMSA burden (dimensions 1, 2, and 3). The association of ATN with MMSE, age, and WMSA burden, as well as the ATN distribution observed in the highly selective homogeneous ADNI cohort suggests that the 
ATN classification scheme may be useful to assess disease staging. The capacity of the AD subtype to drive a dimension by itself, partially independent of ATN and demographic and clinical factors, suggests that $\mathrm{AD}$ subtype classification may be less influenced by disease staging. Whether AD subtypes reflect disease staging or truly distinct subtypes is an open discussion $[2,4,33,48]$ that can only be answered in future longitudinal studies. The distinct subtype hypothesis postulates that there are different pathophysiological pathways underlying clinical syndrome in $\mathrm{AD}[2,5]$. Current data show that these pathways seem to rely on different forms of spread of pathology across the brain $[5,33]$, leading to different patterns of brain atrophy in structural MRI [34]. An advantage of the $\mathrm{AD}$ subtype classification is the inclusion of the topographical dimension to the $\mathrm{N}$ category of ATN [36]. Whether the AD subtype is a stronger approach to disentangle disease heterogeneity than the ATN classification scheme must be confirmed in future studies.

The current study has some limitations. We did not include A- individuals in the main analysis. Including A- individuals might increase the heterogeneity and show slightly different associations between the AD subtype, ATN profiles, and demographic and clinical factors. Further, the methods to assess WMSA were different in $\mathrm{ADNI}$ and KIDS. In the ADNI cohort, we used an automatic segmentation based on white matter hypointensities, while in the KIDS cohort, we used visual ratings based on white matter hyperintensities. Although using different methods for WMSA could induce some noise in our analysis, we recently showed that both methods are strongly associated with each other [28]. Further, by classifying the output from both methods into high and low WMSA burden, we used a rougher measure that is less influenced by differences between the 2 methods and has greater clinical applicability [46]. Finally, we lacked data for several factors listed in the recent conceptual framework for biological subtypes of $\mathrm{AD}$, including disease duration [5]. Future studies should thus extend our current analysis by including measures of education or cognitive reserve, other markers of CVD, information about disease onset or disease duration, and data on specific cognitive domains. Investigating the contribution of other comorbid brain pathologies such as Lewy body pathology or TDP-43 is challenging at present by the lack of reliable biomarkers for these 2 pathologies.

We conclude that the distribution of $\mathrm{AD}$ subtypes and ATN profiles depends on the source of the patients, and it aligns with different demographic and clinical factors, depending on whether the cohort is more selective and ho- mogeneous or more naturalistic and heterogeneous. Our findings largely support the recent conceptual framework of biological subtypes of AD. This framework postulates that the combination of risk factors, protective factors, and comorbid brain pathologies will determine belonging of $\mathrm{AD}$ patients to distinct biological subtypes of $\mathrm{AD}$. Future studies should continue testing this framework with the goal of advancing our currently limited possibilities to realise precision medicine in clinical routine.

\section{Acknowledgements}

ADNI is funded by the National Institute on Aging, the National Institute of Biomedical Imaging and Bioengineering, and several generous contributions from the following: the Alzheimer's Association, the Alzheimer's Drug Discovery Foundation, BioClinica Inc., Biogen Idec Inc., Bristol-Myers Squibb Co., Eisai, Inc., Elan Pharmaceuticals, Inc., Eli Lilly and Co., F. Hoffmann-La Roche Ltd and its affiliated company Genentech Inc., GE Healthcare, Innogenetics NV, IXICO Ltd., Janssen Alzheimer Immunotherapy Research \& Development LLC, Johnson \& Johnson Pharmaceutical Research \& Development LLC, Medpace Inc., Merck \& Co. Inc., Meso Scale Diagnostics LLC, NeuroRx Research, Novartis Pharmaceuticals Corp., Pfizer Inc., Piramal Imaging, Servier, Synarc Inc., and Takeda Pharmaceutical Company. The Canadian Institutes of Health Research is providing funds to support ADNI clinical sites in Canada. Private sector contributions are facilitated by the Foundation for the National Institutes of Health (www.fnih. org). The grantee organization is the Northern California Institute for Research and Education, and the study is coordinated by the Alzheimer's Disease Cooperative Study at the University of California, San Diego. Data collection and sharing for in ADNI were funded by the National Institutes of Health (NIH) (Grant U01 AG024904). ADNI data are disseminated by the Laboratory for Neuro Imaging at the University of California, Los Angeles. This research was also supported by NIH Grants P30 AG010129 and K01 AG030514. These sponsors did not have any involvement in the study design; collection, analysis, and interpretation of data; writing of the report; and the decision to submit the article for publication.

\section{Statement of Ethics}

Written informed consent was obtained from all the patients or a legal guardian, in accordance with the Declaration of Helsinki. For ADNI, study protocols were approved by the Institutional Review Boards at each participating centre. For the Karolinska Imaging Dementia Study (KIDS), ethical approval was obtained from the RegionalEthics Board in Stockholm, Sweden (DNR: 2011/198731/4).

\section{Conflict of Interest Statement}

The authors declare no conflict of interest. 


\section{Funding Sources}

This study was supported by the Swedish Foundation for Strategic Research (SSF), the Strategic Research Programme in Neuroscience at Karolinska Institutet (StratNeuro), the Swedish Research Council (VR, 2016-02282), the regional agreement on medical training and clinical research (ALF) between Stockholm County Council and Karolinska Institutet; Center for Innovative Medicine (CIMED); Demensförbundet, the Swedish Alzheimer Foundation; the Swedish Brain Foundation; the Åke Wiberg Foundation; Demensfonden; Stiftelsen Olle Engkvist Byggmästare; and Birgitta och Sten Westerberg.

\section{Author Contributions}

N.C., D.F., and E.W. contributed to the conception and design of the study. N.C., K.P., S.S., L.C., T.G., S.M., and L.O.W. contributed to the acquisition and analysis of data. N.C., U.E., D.F., and E.W. contributed to drafting a significant portion of the manuscript and preparing the figures. All the authors revised the manuscript and contributed on scientific content.

\section{Availability of Data and Materials}

The datasets generated and analysed during the current study are available in the ADNI data repository, http://adni.loni.usc.edu/ data-samples/access-data/ and at the Karolinska Imaging Dementia Study (KIDS).

\section{References}

1 Veitch DP, Weiner MW, Aisen PS, Beckett LA, Cairns NJ, Green RC, et al. Understanding disease progression and improving $\mathrm{Alz}$ heimer's disease clinical trials: recent highlights from the Alzheimer's disease neuroimaging Initiative. Alzheimer's Dement. 2019; 15:106-52.

2 Ferreira D, Pereira JB, Volpe G, Westman E. Subtypes of Alzheimer's disease display distinct network abnormalities extending beyond their pattern of brain atrophy. Front Neurol. 2019 May;10(May):524.

3 Byun MS, Kim SE, Park J, Yi D, Choe YM, Sohn BK, et al. Heterogeneity of regional brain atrophy patterns associated with distinct progression rates in Alzheimer's disease. PLoS One. 2015;10(11):e0142756-16.

4 Ferreira D, Verhagen C, Hernández-Cabrera JA, Cavallin L, Guo CJ, Ekman U, et al. Distinct subtypes of Alzheimer's disease based on patterns of brain atrophy: Longitudinal trajectories and clinical applications. Sci Rep. 2017;7(March):46263.

5 Ferreira D, Nordberg A, Westman E. Biological subtypes of Alzheimer disease: a systematic review and meta-analysis. Neurology. 2020 Mar;94(10):436-48.

6 Ekman U, Ferreira D, Westman E. The A/T/N biomarker scheme and patterns of brain atrophy assessed in mild cognitive impairment. Sci Rep. 2018 Dec;8(1):8431.

7 Jack CR, Bennett DA, Blennow K, Carrillo MC, Feldman HH, Frisoni GB, et al. A/T/N: an unbiased descriptive classification scheme for Alzheimer disease biomarkers. Neurology. 2016;87(5):539-47.

8 Kandel BM, Avants BB, Gee JC, McMillan CT, Erus G, Doshi J, et al. White matter hyperintensities are more highly associated with preclinical Alzheimer's disease than imaging and cognitive markers of neurodegeneration. Alzheimers Dement. 2016;4:18-27.

9 Lee S, Viqar F, Zimmerman ME, Narkhede A, Tosto G, Benzinger TLS, et al. White matter hyperintensities are a core feature of Alzheimer's disease: evidence from the dominantly inherited Alzheimer network. Ann Neurol. 2016 Jun;79(6):929-39.

10 Wardlaw JM, Smith EE, Biessels GJ, Cordonnier C, Fazekas F, Frayne R, et al. Neuroimaging standards for research into small vessel disease and its contribution to ageing and neurodegeneration. Lancet Neurol. 2013 Aug; 12(8):822-38.

11 Lindemer ER, Greve DN, Fischl BR, Augustinack JC, Salat DH, Neuroimaging D. NeuroImage : clinical regional staging of white matter signal abnormalities in aging and Alzheimer's disease. NeuroImage Clin. 2017;14: 156-65.

12 Ferreira D, Hansson O, Barroso J, Molina Y, Machado A, Hernández-Cabrera JA, et al. The interactive effect of demographic and clinical factors on hippocampal volume: a multicohort study on 1958 cognitively normal individuals. Hippocampus. 2017 Jun; 27(6):653-67.

13 Mueller SG, Weiner MW, Thal LJ, Petersen RC, Jack CR, Jagust W, et al. Ways toward an early diagnosis in Alzheimer's disease: the Alzheimer's disease neuroimaging initiative (ADNI). Alzheimer's Dement.. 2005 Jul;1(1): 55-66.

14 Shams S, Martola J, Granberg T, Li X, Shams M, Fereshtehnejad SM, et al. Cerebral microbleeds: different prevalence, topography, and risk factors depending on dementia diagnosis-the Karolinska imaging dementia study. Am J Neuroradiol. 2015;36(4):661-6.

15 Petersen RC, Aisen PS, Beckett LA, Donohue MC, Gamst AC, Harvey DJ, et al. Alzheimer's disease neuroimaging initiative (ADNI): clinical characterization. Neurology. 2010;74(3): 201-9.

16 Mckhann GM, Knopman DS, Chertkow H, Hyman BT, Jack CR, Kawas CH, et al. The diagnosis of dementia due to Alzheimer's disease : recommendations from the national in- stitute on aging-Alzheimer's association workgroups on diagnostic guidelines for Alzheimer's disease. Alzheimer's Dement. 2011;7(3):263-9.

17 Simmons A, Westman E, Muehlboeck S, Mecocci P, Vellas B, Tsolaki M, et al. The AddNeuroMed framework for multi-centre MRI assessment of Alzheimer's disease: Experience from the first 24 months. Int J Geriatr Psychiatry. 2011;26(1):75-82.

18 Shaw LM, Vanderstichele H, Knapik-Czajka M, Clark CM, Aisen PS, Petersen RC, et al. Cerebrospinal fluid biomarker signature in Alzheimer's disease neuroimaging initiative subjects. Ann Neurol. 2009;65(4):403-13.

19 Shams S, Granberg T, Martola J, Li X, Shams M, Fereshtehnejad SM, et al. Cerebrospinal fluid profiles with increasing number of cerebral microbleeds in a continuum of cognitive impairment. J Cereb Blood Flow Metab. 2016; 36(3):621-8.

20 Muehlboeck JS, Westman E, Simmons A. The HiveDB image data management and analysis framework. Front Neuroinform. 2014;7(January):49-13.

21 Pai A, Sørensen L, Darkner S, Sporring J, Rostrup E, Nielsen M. Ic-p-131: white matter hypointensity growth rate correlates with rate of brain atrophy. Alzheimer's Dementia. 2014 Jul;10(4):P75-6.

22 Schiffmann R, Van Der Knaap MS. Invited article: an MRI-based approach to the diagnosis of white matter disorders. Neurology. 2009; 72(8):750-9.

23 Ferreira D, Voevodskaya O, Imrell K, Stawiarz L, Spulber G, Wahlund LO, et al. Multiple sclerosis patients lacking oligoclonal bands in the cerebrospinal fluid have less global and regional brain atrophy. J Neuroimmunol. 2014; 274(1-2):149-54.

24 Narayana PA, Zhou Y, Hasan KM, Datta S, Sun X, Wolinsky JS. Hypoperfusion and T1hypointense lesions in white matter in multiple sclerosis. Mult Scler. 2014;20(3):365-73. 
25 Leritz EC, Shepel J, Williams VJ, Lipsitz LA, Mcglinchey RE, Milberg WP, et al. Associations between $\mathrm{T} 1$ white matter lesion volume and regional white matter microstructure in aging. Hum Brain Mapp. 2015;35(3):1085100.

26 Fazekas F, Chawluk JB, Zimmerma A, June M. MR signal abnormalities at $1.5 \mathrm{~T}$ in Alzheimer's dementia and normal aging deficiency. AJR Am J Roentgenol. 1987;149(August):351-6.

27 Kapeller P, Barber R, Vermeulen RJ, Adèr $H$, Scheltens $P$, Freidl W, et al. Visual rating of age-related white matter changes on magnetic resonance imaging: scale comparison, interrater agreement, and correlations with quantitative measurements. Stroke. 2003; 34(2):441-5.

28 Cedres N, Ferreira D, Machado A, Shams S, Sacuiu S, Waern M, et al. Predicting Fazekas scores from automatic segmentations of white matter signal abnormalities. Aging. 2020 Jan;12(1):894-901.

29 Ferreira D, Cavallin L, Larsson EM, Muehlboeck JS, Mecocci P, Vellas B, et al. Practical cut-offs for visual rating scales of medial temporal, frontal and posterior atrophy in $\mathrm{Alz}$ heimer's disease and mild cognitive impairment. J Intern Med. 2015 Sep;278(3):277-90.

30 Scheltens P, Leys D, Barkhof F, Huglo D, Weinstein HC, Vermersch P, et al. Atrophy of medial temporal lobes on MRI in "probable" Alzheimer's disease and normal ageing: diagnostic value and neuropsychological correlates. J Neurol Neurosurg Psychiatry. 1992;55: 967-72.

31 Koedam EL, Lehmann M, van der Flier WM, Scheltens P, Pijnenburg YA, Fox N, et al. Visual assessment of posterior atrophy development of a MRI rating scale. Eur Radiol. 2011 Dec;21(12):2618-25.
32 Ferreira D, Cavallin L, Granberg T, Lindberg $\mathrm{O}$, Aguilar C, Mecocci P, et al. Quantitative validation of a visual rating scale for frontal atrophy: associations with clinical status, APOE e4, CSF biomarkers and cognition. Eur Radiol. 2016 Aug;26(8):2597-610.

33 Murray ME, Graff-Radford NR, Ross OA, Petersen RC, Duara R, Dickson DW. Neuropathologically defined subtypes of Alzheimer's disease with distinct clinical characteristics: a retrospective study. Lancet Neurol. 2011 Sep;10(9):785-96.

34 Whitwell JL, Dickson DW, Murray ME, Weigand SD, Tosakulwong N, Senjem ML, et al. Neuroimaging correlates of pathologically defined subtypes of Alzheimer's disease: a case-control study. Lancet Neurol. 2012 Oct; 11(10):868-77.

35 Persson K, Eldholm RS, Barca ML, Cavallin L, Ferreira D, Knapskog AB, et al. MRI-assessed atrophy subtypes in Alzheimer's disease and the cognitive reserve hypothesis. PLoS One. 2017;12(10):e0186595-15.

36 Ferreira D, Shams S, Cavallin L, Viitanen M, Martola J, Granberg T, et al. The contribution of small vessel disease to subtypes of Alzheimer's disease: a study on cerebrospinal fluid and imaging biomarkers. Neurobiol Aging. 2018; 70:18-29.

37 Poulakis K, Pereira JB, Mecocci P, Vellas B, Tsolaki M, Kłoszewska I, et al. Heterogeneous patterns of brain atrophy in Alzheimer's disease. Neurobiol Aging. 2018;65:98-108.

38 Lê S, Josse J, Husson F. FactoMineR : an R package for multivariate analysis. J Stat Softw. 2008 Feb;25(1):253-8.
39 Stekhoven DJ, Bühlmann P. MissForest-nonparametric missing value imputation for mixed-type data. Bioinformatics. 2012;28(1): $112-8$.

40 Kassambara A, Mundt F. Package 'factoextra' R topics documented . 2020;84

41 Husson AF, Josse J, Le S, Mazet J, Husson MF. Package 'FactoMineR'. 2020

42 Hochberg Y, Benjamini Y. More powerful procedures for multiple significance testing. Stat Med. 1990 Jul;9(7):811-8.

43 Jack CR, Bennett DA, Blennow K, Carrillo MC, Dunn B, Haeberlein SB, et al. NIA-AA research framework: toward a biological definition of Alzheimer's disease. Alzheimer's Dement.. 2018;14(4):535-62.

44 Lechner H, Schmidt R, Bertha G, Justich E, Offenbacher H, Schneider G. Nuclear magnetic resonance image white matter lesions and risk factors for stroke in normal individuals. Stroke. 1988;19(2):263-5.

45 Debette S, Markus HS. The clinical importance of white matter hyperintensities on brain magnetic resonance imaging: systematic review and meta-analysis. BMJ. 2010; 341(7767):c3666

46 Hilal S, Mok V, Youn YC, Wong A, Ikram MK, Chen CL. Prevalence, risk factors and consequences of cerebral small vessel diseases: data from three Asian countries. J Neurol Neurosurg Psychiatry. 2017;88(8):669-74.

47 Kanekiyo T, Xu H, Bu G. ApoE and A $\beta$ in Alzheimer's disease: accidental encounters or partners? Neuron. 2014 Feb;81(4):740-54.

48 Zhang X, Mormino EC, Sun N, Sperling RA, Sabuncu MR, Yeo BT. Bayesian model reveals latent atrophy factors with dissociable cognitive trajectories in Alzheimer's disease. Proc Natl Acad Sci USA. 2016 Oct;113(42):E6535E6544. 